\title{
Statistical mechanics of finite arrays of coupled bistable elements
}

\author{
J. Gómez-Ordóñez ${ }^{1}$, J. M. Casado ${ }^{1}$, M. Morillo $^{1(a)}, \mathrm{C}^{\left(\mathrm{HonisCH}^{2}\right.}$ and R. Friedrich ${ }^{2}$ \\ ${ }^{1}$ Física Teórica, Universidad de Sevilla - Apartado de Correos 1065, Sevilla 41080, Spain, EU \\ ${ }^{2}$ Institut für Theoretische Physik, Westfälische Wilhelms-Universität Münster - D-48149 Münster, Germany, EU
}

received 3 July 2009; accepted in final form 30 October 2009

published online 27 November 2009

PACS 05.45.Xt - Synchronization; coupled oscillators

PACS 05.10.Gg - Stochastic analysis methods (Fokker-Planck, Langevin, etc.)

PACS $05.40 . \mathrm{Ca}$ - Noise

\begin{abstract}
We discuss the equilibrium of a single collective variable characterizing a finite set of coupled, noisy, bistable systems as the noise strength, the size and the coupling parameter are varied. We identify distinct regions in parameter space. Our finite size results indicate important qualitative differences with respect to those obtained in the asymptotic infinite size limit. We also discuss how to construct approximate 1-dimensional Langevin equations. This equation provides a useful tool to understand the collective behavior even in the presence of an external driving force.
\end{abstract}

Copyright (C) EPLA, 2009

Introduction. - Stochastic resonance $(\mathrm{SR})$ is a phenomenon where the response of a nonlinear dynamical system to an external driving is enhanced by the action of noise [1]. Although SR has been mainly discussed for simple systems, its analysis has also been extended to complex systems with many interacting units [2-6]. Our present study is prompted by recent studies on SR effects on the collective variable of finite arrays [7-10]. Typically, the noise strength is the parameter varied to observe SR effects [1]. In arrays, the coupling strength $[5,8]$, as well as the system size [6,7] have also been considered as parameters leading to SR. The complexity of the SR analysis of the collective variable characterizing the array can be facilitated by an adequate understanding of the equilibrium state. In this work, we carry out a detailed numerical analysis of the equilibrium distribution of the collective variable. On the other hand, the reduction of the multidimensional Langevin dynamics of the array degrees of freedom to an effective 1-dimensional Langevin equation greatly simplifies the analysis of the system response to weak driving forces. Here we will assess the possibility of such a reduced Langevin description.

The model. - We consider a set of $N$ identical bistable units, each of them characterized by a variable $x_{i}(t)(i=$ $1, \ldots, N)$ satisfying a stochastic evolution equation (in dimensionless form) of the type $[7,11,12]$

$$
\dot{x}_{i}=x_{i}-x_{i}^{3}+\frac{\theta}{N} \sum_{j=1}^{N}\left(x_{j}-x_{i}\right)+\sqrt{2 D} \xi_{i}(t),
$$

(a) E-mail: morillo@us.es where $\theta$ is a coupling parameter and the term $\xi_{i}(t)$ represents a Gaussian white noise with zero average and $\left\langle\xi_{i}(t) \xi_{j}(s)\right\rangle=\delta_{i j} \delta(t-s)$. The set $x_{i}(t)$ is an $N$-dimensional Markovian process.

An alternative formulation of the dynamics is in terms of the Fokker-Planck equation for the joint probability density $f_{N}\left(x_{1}, \ldots, x_{N}, t\right)$,

$$
\frac{\partial f_{N}}{\partial t}=\sum_{i} \frac{\partial}{\partial x_{i}}\left(\frac{\partial U}{\partial x_{i}} f_{N}\right)+D \sum_{i} \frac{\partial^{2} f_{N}}{\partial x_{i}^{2}}
$$

where $U$ is the potential energy relief,

$$
U\left(x_{1}, \ldots, x_{N}\right)=\sum_{i=1}^{N} V\left(x_{i}\right)+\frac{\theta}{4 N} \sum_{i=1}^{N} \sum_{j=1}^{N}\left(x_{i}-x_{j}\right)^{2}
$$

with

$$
V(x)=\frac{x^{4}}{4}-\frac{x^{2}}{2} .
$$

We are interested in the properties of a collective variable,

$$
S(t)=\frac{1}{N} \sum_{j} x_{j}(t) .
$$

Even though the set $x_{i}(t)$ is a Markovian process, $S(t)$ in general is not. Consequently, there is no reason why there should exist a Langevin description of $S(t)$ or the corresponding probability density $P(s, t)$ satisfy a FokkerPlanck equation. 
The equilibrium distribution for the collective variable. - The joint equilibrium probability distribution $f^{\text {eq }}\left(x_{1}, \ldots, x_{n}\right)$ is the stationary solution of eq. (2):

$$
f^{\mathrm{eq}} \propto \exp \left(-\frac{U}{D}\right) .
$$

The equilibrium probability density of the collective variable, $P^{\mathrm{eq}}(s)$, is defined as

$$
\begin{aligned}
P^{\mathrm{eq}}(s)= & \langle\delta(s-S(t))\rangle^{\mathrm{eq}}= \\
& \int \mathrm{d} x_{1} \cdots \mathrm{d} x_{N} \delta\left(s-\frac{1}{N} \sum_{j} x_{j}(t)\right) f^{\mathrm{eq}} .
\end{aligned}
$$

In the limit $N \rightarrow \infty$, Desai and Zwanzig [11] carried out an asymptotic analysis of eq. (7) based on a saddle point approximation and obtained formal expressions for $P_{\infty}^{\mathrm{eq}}(s)$. In their analysis, Desai and Zwanzig introduced a convenient parameter $z=\frac{|\theta-1|}{\sqrt{2 D}}$ and found that there is a critical line in a $z$-vs. $-\theta$ diagram. At this critical line, the asymptotic probability distribution bifurcates. The nature of this bifurcation is such that for $z$ values below the critical line, there is a single stable distribution, symmetrical around $s=0$, and either bimodal (for $\theta<1$ ) or monomodal (for $\theta>1$ ). On the other hand, for $z$ values above the critical line, there are two distinct stable monomodal distributions centered around $\pm s_{0} \neq 0$. Whether the system relaxes to one of the stable equilibrium solutions depends on its initial preparation. Clearly, this bifurcation of the probability density implies a bifurcation of the average value $\langle s\rangle_{\infty}^{\text {eq }}$. Further details about the statistical mechanical description of the system in the $N \rightarrow \infty$ limit were discussed later by other authors [12-18].

For finite $N$, an exact analytical evaluation of the multidimensional integral in eq. (7) is not feasible. Nonetheless, the symmetry of the potential relief given in eq. (3) guarantees that the equilibrium average value $\langle s\rangle_{\mathrm{eq}}=0$ for any values of the parameters. Furthermore, the $H$-theorem for finite systems guarantees [19] that the equilibrium distribution function of the $N$-dimensional $x_{i}(t)$ process is unique regardless of the initial condition. Thus, according to its definition in eq. (7), there is a unique stable $P^{\mathrm{eq}}(s)$ and the possibility of a bifurcation of $P^{\mathrm{eq}}(s)$ and for any of its moments for finite $N$ is ruled out.

It is then clear that the $N \rightarrow \infty$ analysis is inadequate to describe the behavior of systems with a finite number of elements. To proceed we turn to numerical simulations. We numerically integrate the set of equations in eq. (1) for very many realizations of the noise terms using the procedure detailed in [20]. After a transient period we start gathering data and average over the noise realizations to construct histograms estimating $P^{\mathrm{eq}}(s)$. We also evaluate the equilibrium time correlation function of the collective variable and check that the system has indeed relaxed to an equilibrium situation.

Our numerical findings indicate that, depending on the $D$ and $\theta$ values, the equilibrium probability density takes

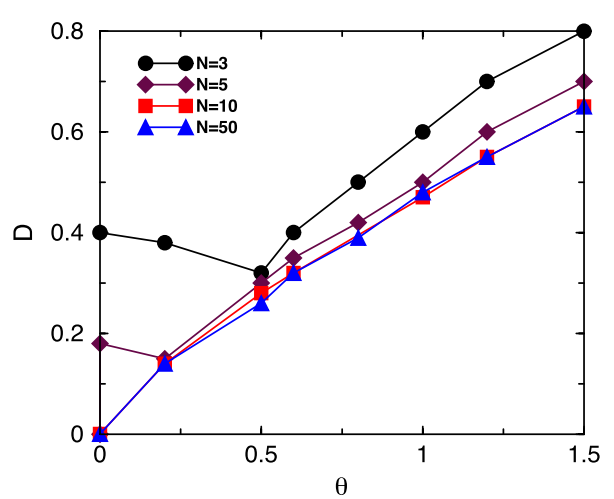

Fig. 1: (Color online) Numerically determined lines separating different regions in parameter space. The equilibrium density $P^{\mathrm{eq}}(s)$ is monomodal above the line and multimodal below it. Circles correspond to a system with $N=3$, diamonds to $N=5$, squares to $N=10$ and triangles to $N=50$.

different shapes. In fig. 1 we show results obtained for systems with $N=3,5,10,50$ depicting transition lines separating regions in $D$-vs. $-\theta$ plots. For parameter values above the corresponding line, the equilibrium probability density is symmetrical around a single maximum at $s=0$. For parameter values below the lines, the distribution is also symmetrical around its minimum at $s=0$ with several maxima located at $s \neq 0$. The change in the shape of $P^{\text {eq }}(s)$ as the parameters $\theta$ or $D$ are varied is a gradual one. Indeed, the values for $\theta$ and $D$ depicted in fig. 1 correspond to situations where the probability density presents a rather broad maximum around $s=0$.

For $\theta=0$, we have independent subsystems and even for moderate $N$, the central limit theorem indicates that a Gaussian-like shape for $P^{\mathrm{eq}}(s)$ is expected. This is why the transition $D$ value in fig. 1 is very small as $\theta \rightarrow 0$ for $N=10,50$. On the other hand, for very small systems (see results for $N=3,5$ ), deviations from the central limit theorem might be large and $P^{\mathrm{eq}}(s)$ might be very different from a Gaussian, unless the noise strength is large enough. Then the behavior for $N=3,5$ depicted in fig. 1 for $\theta=0$ is not surprising. When $\theta \neq 0$, the central limit theorem does not strictly apply and it is only for sufficiently large $D$ values that $P^{\mathrm{eq}}(s)$ is monomodal. Qualitatively, the numerical results can be rationalized by noting that the transitions among barriers in the $N$-dimensional energy surface are induced by the noise. Then, for large noise strengths, the random trajectories of $x_{i}(t)$ have good chances of exploring quite frequently all the attractors with numerous jumps over the barriers, leading to a single maximum distribution for the global variable. For low noise strengths the bimodality of the distribution is to be expected due to the rather scarce number of jumps over high energy regions.

It should be noted that the depicted lines are not critical lines or bifurcation lines. Actually, it would be perhaps more appropriate to talk about a transition region where the shape of the distribution changes gradually between 


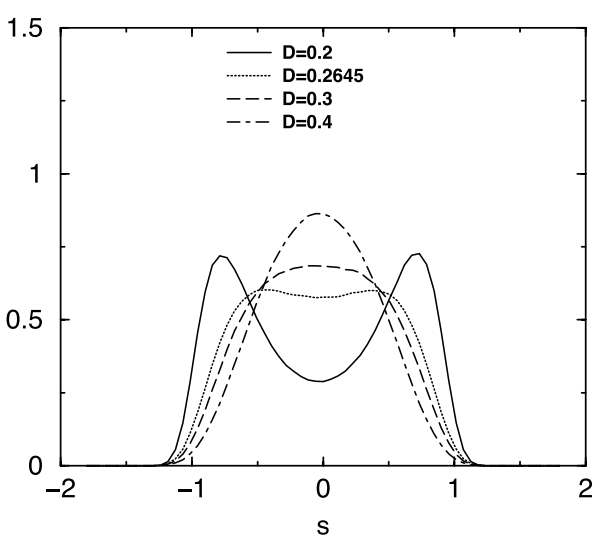

Fig. 2: $P^{\mathrm{eq}}(s)$ for a $N=10$ units system with $\theta=0.5$ and several values of $D$, obtained from the simulations of the full set of equations, eq. (1).

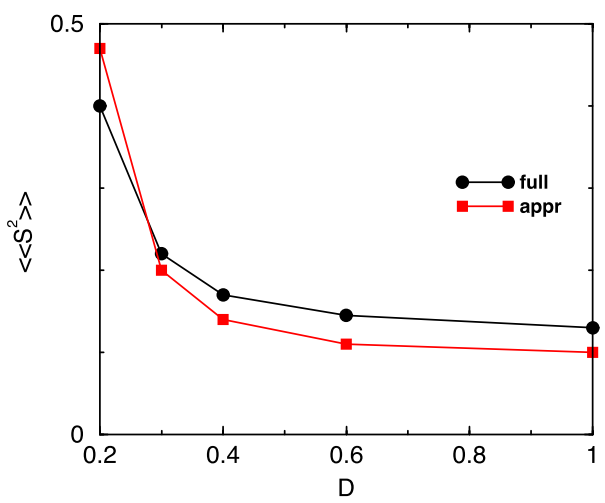

Fig. 3: (Color online) Circles correspond to equilibrium values of the second cumulant $\left\langle\left\langle S^{2}\right\rangle\right\rangle_{\infty}$ for $N=10, \theta=0.5$ and several values of $D$, obtained from the simulations of the full set of equations, eq. (1). The squares correspond to the second cumulant $\left\langle\left\langle S^{2}\right\rangle\right\rangle_{\infty}^{\text {appr }}$ obtained from the numerical simulations of the approximation given by eq. (8) using the same parameter values.

monomodal and multimodal. An example of this gradual change of shape of $P^{\mathrm{eq}}(s)$ as $D$ is varied with $\theta$ kept constant can be seen in fig. 2 for $\theta=0.5$ and $N=10$. The average value $\langle S\rangle_{\infty}=0$ for all values of $D$ and $\theta$, while the second cumulant of the distribution, $\left\langle\left\langle S^{2}\right\rangle\right\rangle_{\infty}=$ $\left\langle S^{2}\right\rangle_{\infty}-\langle S\rangle_{\infty}^{2}$ also varies gradually across the transition region as depicted in fig. 3 for $\theta=0.5$ and $N=10$.

These facts are in very sharp contrast with the behavior expected in the $N \rightarrow \infty$ limit. It is only in this limit that a change in values of the noise strength or the coupling constant might yield a bifurcation of the probability distribution or its moments. For finite systems no bifurcation exists as any of those two parameter values are varied, as expected from theoretical considerations and corroborated by our numerical analysis.

It should be pointed out that the shape of $P^{\mathrm{eq}}(s)$ does not imply the shape for the one-particle equilibrium distribution function $f_{1}^{\mathrm{eq}}(x)$ formally obtained by integrating the joint equilibrium probability distribution over all the

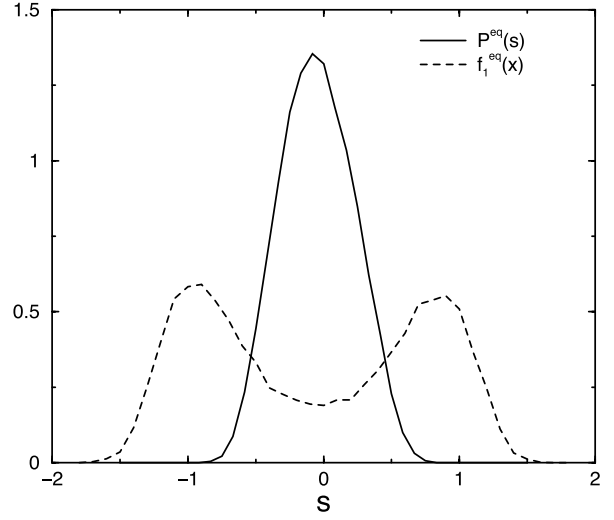

Fig. 4: Plots of the equilibrium distribution for the collective variable $P^{\mathrm{eq}}(s)$ and the equilibrium distribution for one of the subsystems, $f_{1}^{\text {eq }}(x)$, for a system with $N=50$ units coupled with a coupling strength $\theta=0.2$ and with $D=0.15$.

degrees of freedom except one. Histograms estimating $f_{1}^{\text {eq }}(x)$ can also be constructed from our numerical simulations. An example is shown in fig. 4, where we note that for the parameter values $N=50, D=0.15$, and $\theta=0.2$, $P^{\mathrm{eq}}(s)$ is monomodal, while $f_{1}^{\mathrm{eq}}(x)$ is bimodal.

A few years ago, Pikovsky et al. [7] proposed an approximate description of the model with finite $N$ in terms of the collective variable, $S$, and a new variable defined as $M=(1 / N) \sum_{i}\left(x_{i}-S\right)^{2}$. They are assumed to obey the stochastic equations

$$
\begin{aligned}
\dot{S} & =(1-3 M) S-S^{3}+\sqrt{\frac{2 D}{N}} \eta(t), \\
\frac{1}{2} \dot{M} & =\left(1-\theta-3 S^{2}\right) M-3 M^{2}+D,
\end{aligned}
$$

where $\eta(t)$ is a Gaussian white noise with zero mean and $\langle\eta(t) \eta(s)\rangle=\delta(t-s)$. The nonlinearity of these equations preclude their exact analytical treatment. We have carried out numerical simulations of these two equations. After averaging over a large number of noise realizations we evaluate the long time noise average values of the variables. We find that $\langle S\rangle^{\text {appr }} \rightarrow 0$ in agreement with the exact result. In fig. 3 we depict the results for $\left\langle\left\langle S^{2}\right\rangle\right\rangle^{\text {appr }}$, obtained from the long time numerical solutions of eq. (8) and $\left\langle\left\langle S^{2}\right\rangle\right\rangle_{\infty}$ obtained from the numerical simulation of eq. (3). The data correspond to $N=10$ and $\theta=0.5$. There are significant discrepancies between the numerical results obtained from the approximate set and those corresponding to the simulations of the full set of equations. Similar discrepancies happen for other values of $N$ and $\theta$. Nonetheless, the Gaussian approximation is a reasonable good one.

Effective 1-dimensional Langevin dynamics for the collective variable. - There have been several attempts to construct effective 1-dimensional Langevin dynamics for the collective variable for finite systems $[7,10]$. Although such an equation does not necessarily 
exist as $S(t)$ is not necessarily a Markovian process, if it does, it might be a useful and reliable approximation.

In [7], Pikovsky et al. applied a slaving principle to reduce the dynamics in eq. (8) to an effective 1-dimensional Langevin equation,

$$
\dot{S}=a S-b S^{3}+\sqrt{\frac{2 D}{N}} \eta(t) .
$$

The coefficients $a$ and $b$ are given by

$$
\begin{aligned}
a & =1+0.5(\theta-1)-0.5 \sqrt{(\theta-1)^{2}+12 D} \\
b & =\frac{4 a}{2-\theta+\sqrt{(2+\theta)^{2}-24 D}} .
\end{aligned}
$$

Unfortunately, $b$ may be a complex number for some values of $D$ and $\theta$.

The method described in $[21,22]$ opens up another possibility to construct an effective 1-dimensional Langevin equation by numerically estimating the Kramers-Moyal coefficients $D^{(n)}(S, t)$ for a given data set of a general process $S(t)$. These coefficients are defined as

$$
D^{(n)}(S, t)=\left.\frac{1}{n !} \lim _{\tau \rightarrow 0} \frac{1}{\tau}\left\langle(S(t+\tau)-S)^{n}\right\rangle\right|_{S(t)=S}
$$

As described in $[21,22]$ these conditional moments can be estimated for the smallest available $\tau$ by computing histograms. Given that the collective variable $S(t)$ is a Markovian process on this scale, i.e., the Markov length is smaller than $\tau$, this procedure yields a reliable 1-dimensional Langevin description of the form

$$
\dot{S}=D^{(1)}(S, t)+\sqrt{2 D^{(2)}(S, t)} \chi(t) .
$$

We find that the fourth coefficient $D^{(4)}$ is about four orders of magnitude smaller than $D^{(2)^{2}}$, i.e., it practically vanishes. Hence, the Pawula theorem guarantees that the third and all higher coefficients do also vanish.

In fig. 5 we depict our numerically estimated $D^{(1)}(S)$ for the system in eq. (1) with $N=10, \theta=0.5$ and varying noise strengths. As the process is stationary, the KramersMoyal coefficients are independent of $t$. The noise value $D_{c} \approx 0.26$ corresponds to the transition point in the $D$-vs.- $\theta$ diagram. The most important result is that one needs polynomials of fifth degree in $S$ to fit the drift coefficient. This shows that the approximations made by Pikovsky, as well as the cumulant expansions presented in [10] should be improved.

Another important finding is that the diffusion coefficient is practically constant and satisfies

$$
D^{(2)}=\frac{D}{N}
$$

This relation is also valid for all other parameters we have tested. While the projection of a high-dimensional stochastic process with additive noise onto a low-dimensional

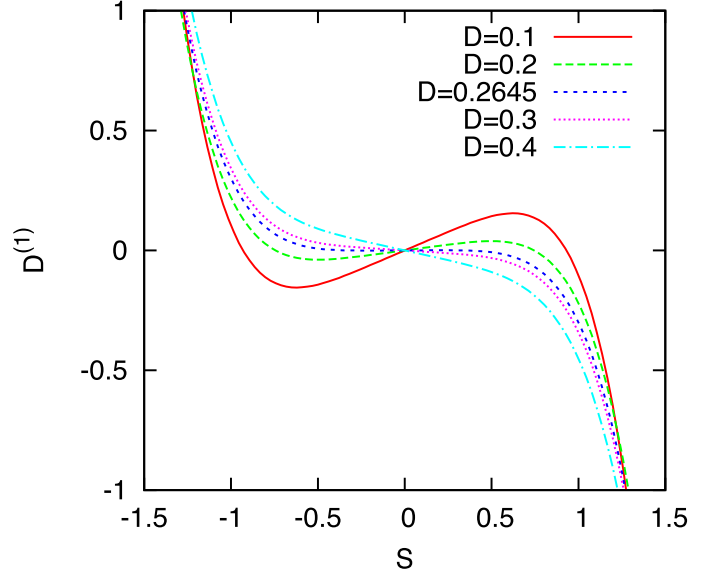

Fig. 5: (Color online) The drift coefficient $D^{(1)}(S)$ for the effective Langevin, eq. (12), for a system described by eq. (1) with $N=10, \theta=0.5$ and several $D$ values.

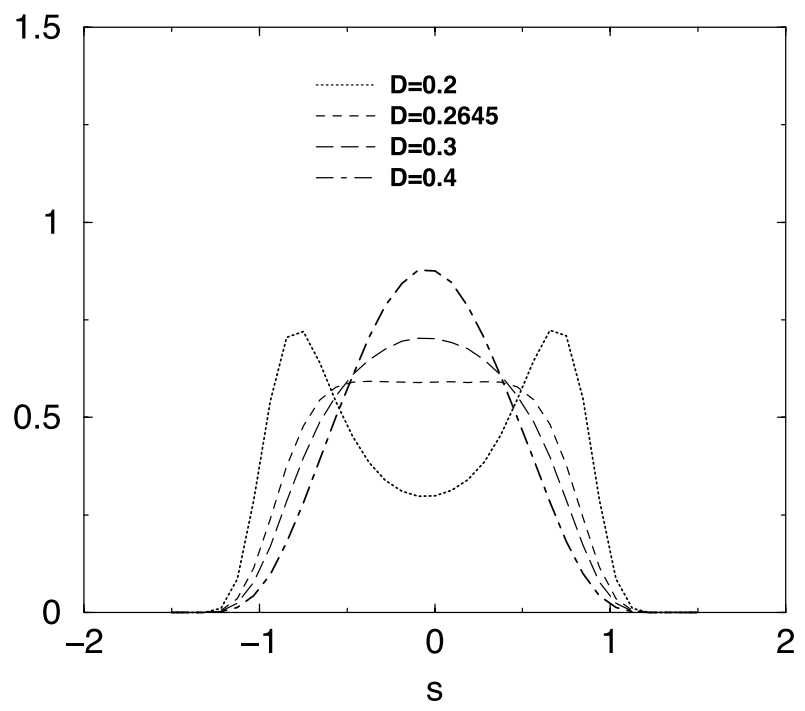

Fig. 6: Equilibrium distribution functions for the collective variable of a $N=10$ units system with coupling parameter $\theta=0.5$ and several $D$ values obtained from the approximate Langevin equation, eq. (12).

subspace usually results in multiplicative noise, we see that the effective noise in the present case remains additive.

We have also generated histograms for the equilibrium distribution using the effective Langevin equation, eq. (12), for parameter values above and below the transition line in fig. 1. The results are shown in fig. 6. Comparing with fig. 2 we see that the effective Langevin equation in eq. (12) reproduces quite correctly the results obtained from the full set of equations for points above and below the transition line.

The enhancement of stochastic resonance for coupled bistable systems was first studied by Jung et al. [2] some years ago. In our recent work [9], we have analyzed the phenomenon of SR for the collective variable of an array driven by weak periodic forces. It was demonstrated that 

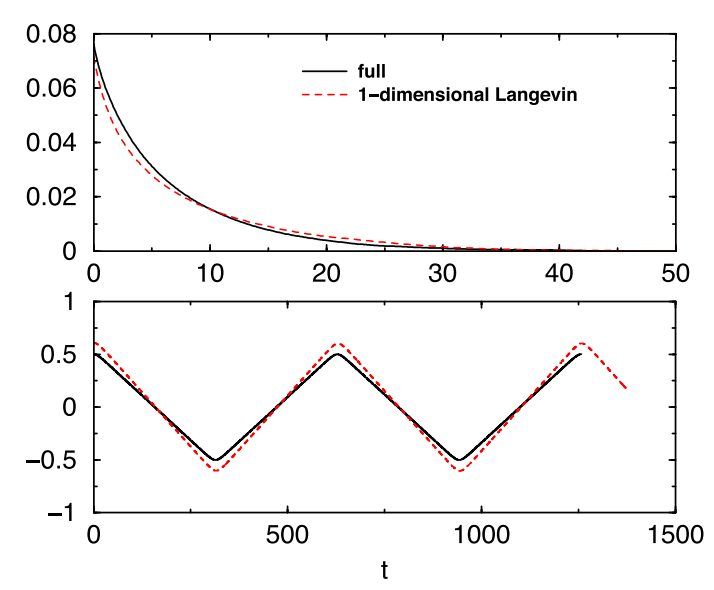

Fig. 7: (Color online) The coherent (lower panel) and incoherent (upper panel) parts of the correlation function of $S(t)$ in a system with $N=10, \theta=0.5, D=0.24$ driven by a dichotomic force with $A=0.1$ and $\Omega=0.01$. The results depicted in black correspond to the simulations of eq. (1), while the red dashed lines are the results obtained with the 1-dimensional Langevin equation in (12).

when the noise strength is varied while keeping $\theta$ and $N$ constant, the signal-to-noise ratio (SNR) of $S(t)$ shows a nonmonotonic behavior with $D$. Even for weak driving amplitudes, the SNR reaches values much larger than those typically observed in single unit systems for the same driving forces. This enhancement is largely due to the strong reduction of the fluctuations in the driven system with respect to those present in the absence of driving. The multidimensional character of the full potential relief makes it difficult to give a simple explanation of the SNR enhancement. The simplicity of the 1-dimensional Langevin equation allows us to understand the reduction of the fluctuations in terms of the drastic differences between the drift coefficient $D^{(1)}(S)$ and $D^{(1)}(S, t)$ in driven systems. For driven systems, eq. (12) also leads to a good approximation to the correlation function as seen in fig. 7 where we depict the results for the incoherent part (upper panel) and the coherent part (lower panel) of the correlation function for $S(t)$ as obtained from the simulation of eq. (1) (solid lines) and the approximate Langevin equation, eqs. (12) and (11) (dashed lines) for $N=10, \theta=0.5, D=0.24$ and a driving dichotomic force with amplitude $A=0.1$ and fundamental frequency $\Omega=0.01 . D^{(2)}$ is well approximated by (13) while $D^{(1)}(S, t)$ is fitted by fifth-order polynomials for each half-period.

Concluding remarks. - In conclusion, we have analyzed a single collective variable characterizing a finite set of noisy bistable units with global coupling. We find several regions in parameters space separated by transition lines. The shape of $P^{\mathrm{eq}}(s)$ switches from monomodal to multimodal as we move across the transition line. Our results show relevant qualitative differences with those obtained in the infinite size limit. We have also found approximate 1-dimensional Langevin descriptions for $S(t)$ valid above and below the transition lines. The change in the shape of $P^{\mathrm{eq}}(s)$ implies the change on the drift coefficient as the values of the noise strength and coupling parameters are modified. In the presence of driving, the corresponding 1-dimensional Langevin equation provides a reliable tool to understand the enhancement of the stochastic resonance effect in arrays relative to the one observed in a single bistable unit.

We acknowledge the support of the Ministerio de Ciencia e Innovación of Spain (FIS2008-04120)

\section{REFERENCES}

[1] Gammaitoni L. et al., Rev. Mod. Phys., 70 (1998) 223.

[2] Jung P., Behn U., Pantazelou E. and Moss F., Phys. Rev. A, 46 (1992) R1909.

[3] Jung P. and Mayer-Kress G., Phys. Rev. Lett., 74 (1995) 2130.

[4] Lindner J. F. et al., Phys. Rev. Lett., 75 (1995) 3.

[5] Schimansky-Geier L. and Siewert U., Stochastic Dynamics (Springer, Berlin) 1997, p. 245.

[6] Neiman A. et al., Phys. Rev. E, 56 (1997) R9.

[7] Pikovsky A., Zaikin A. and de la Casa M. A., Phys. Rev. Lett., 88 (2002) 050601.

[8] Casado J. M. et al., Phys. Rev. E, 73 (2006) 011109.

[9] Morillo M., Gómez-Ordóñez J. and Casado J. M., Phys. Rev. E, 78 (2008) 021109.

[10] Cubero D., Phys. Rev. E, 77 (2008) 021112.

[11] Desai R. C. and Zwanzig R., J. Stat. Phys., 19 (1978) 1.

[12] Dawson D. A., J. Stat. Phys., 31 (1983) 29.

[13] Brey J. J., Casado J. M. and Morillo M., Physica A, 128 (1984) 497.

[14] Shinno M., Phys. Rev. A, 36 (1987) 2393.

[15] Casado J. M. and Morillo M., Phys. Rev. A, 42 (1990) 1875.

[16] Drozdov A. N. and Morillo M., Phys. Rev. E, 54 (1996) 931.

[17] Drozdov A. N. and Morillo M., Phys. Rev. E, 54 (1996) 3304.

[18] Drozdov A. N. and Morillo M., Phys. Rev. Lett., 77 (1996) 3280.

[19] Risken H., The Fokker-Planck Equation (Springer, Berlin) 1984.

[20] Casado-Pascual J., Denk C., Gómez-Ordóñez J., Morillo M. and Hängai P., Phys. Rev. E, 67 (036109) 2003.

[21] Siegert S. et al., Phys. Lett. A, 243 (1998) 275.

[22] Friedrich R. et al., Phys. Lett. A, 271 (2000) 217. 